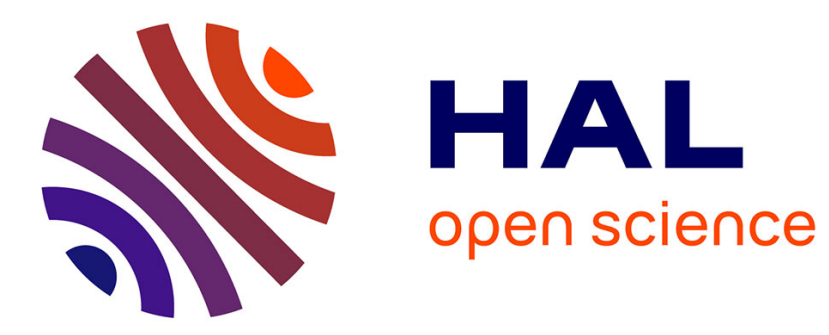

\title{
Modeling and Control of a Convertible Mini-UAV
}

\author{
Duc Anh Ta, Isabelle Fantoni, Rogelio Lozano
}

\section{To cite this version:}

Duc Anh Ta, Isabelle Fantoni, Rogelio Lozano. Modeling and Control of a Convertible Mini-UAV. 18th IFAC World Congress (IFAC WC 2011), Aug 2011, Milan, Italy. pp.1492-1497. hal-00657411

\section{HAL Id: hal-00657411 https://hal.science/hal-00657411}

Submitted on 6 Jan 2012

HAL is a multi-disciplinary open access archive for the deposit and dissemination of scientific research documents, whether they are published or not. The documents may come from teaching and research institutions in France or abroad, or from public or private research centers.
L'archive ouverte pluridisciplinaire HAL, est destinée au dépôt et à la diffusion de documents scientifiques de niveau recherche, publiés ou non, émanant des établissements d'enseignement et de recherche français ou étrangers, des laboratoires publics ou privés. 


\title{
Modeling and Control of a Convertible Mini-UAV
}

\author{
Duc Anh Ta* Isabelle Fantoni ${ }^{*}$ Rogelio Lozano** \\ * UTC-CNRS UMR 6599 HEUDIASYC, 60200 Compiègne, France \\ (e-mail: Duc-Anh.Ta, Isabelle.Fantoni@hds.utc.fr) \\ ** HEUDIASYC and UMI LAFMIA 3175 CNRS-CINVESTAV, \\ Mexico (e-mail: Rogelio.Lozano@hds.utc.fr)
}

\begin{abstract}
The aim of this paper is to present a UAV's configuration that combines the manoeuvrability of a rotary wing vehicle (helicopter) such as slow forward displacement, vertical take off and landing, and the performance of a fixed wing vehicle (plane) such as fast forward movement, long reach and superior endurance.
\end{abstract}

Keywords: Convertible Plane, Modelling, Control, Stabilization and Autonomous Transition.

\section{INTRODUCTION}

In recent years, the interest for Unmanned Aerial Vehicle (UAV) has steadily increased. Aircrafts have been used at first mainly in the military, in reconnaissance, surveillance and also in offensive missions. Nowadays, we can find civilian applications such as traffic monitoring, surveillance and environmental protection, the search and rescue of injured persons or the management of major infrastructure. The purpose of this work is to develop a miniature aircraft which has the performance of a plane for the horizontal flight and the manoeuvrability of a helicopter for the vertical flight. In the past, this research has generated a considerable interest because this type of aircraft doesn't require a runway and the ability of hover makes it very useful for aerial surveillance missions. The most common configuration of convertible aircrafts is the Tail-sitter thanks to its ability of vertical takeoff and landing and the ability of horizontal flight like a conventional plane. Among convertible UAVs in the past, we have been interested in the Heliwing of Boeing and the T-Wing in Stone [2004] of the University of Sydney. The Heliwing was developed in 1995, the T-wing in 2003 and they are both Tail-sitters. Using linear control techniques to stabilize and control the linearized dynamics, the TWing successfully performed the transition from vertical to horizontal and vice versa. However, the Heliwing was lost in an accident during its first transition from the horizontal flight to the vertical flight. Another interesting configuration found in Green et al [2009] which presents a small conventional plane with a capacity of hovering can make the transition from horizontal to vertical. However, this configuration presents a problem related to gyroscopic effect and then the yaw control is not assured when the plane flies vertically. In addition, this configuration can not take off and land vertically. Other simulation results for the transition of a miniature convertible tailsitter UAV using an adaptive quaternion controller and a quaternion gain sequencing feedback controller are presented in Knoebel et al [2008] and Bataillé et al [2008]. However these con-

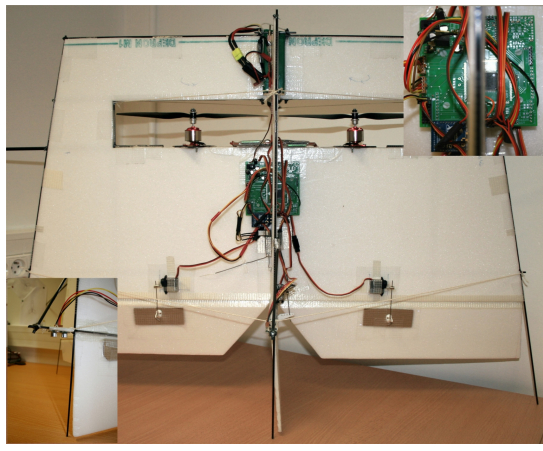

Fig. 1. Prototype of the Convertible Plane

trol laws are high time-consuming and not suitable for embedded control.

This paper contributes to a proposed configuration of the convertible aircraft capable of performing the hovering, transition, vertical take off and landing (figure 1). The detailed mathematical model is obtained by the equations of Newton-Euler and the aerodynamic formulas. In terms of control, we propose a control strategy using the quaternion in order to avoid the singularity problem when the plane is vertical and a motion profile generator to realize the phases of take-off, landing and transition. The paper is organized in five sections. The second section is devoted to the representation of the attitude of the plane. The mechanical structure and the aerodynamic model of convertible aircraft will be presented in the third section. The fourth section contains our contributions, namely the presentation of a control strategy to achieve a longitudinal flight path. Simulation and experimental results are presented in the fifth section. Finally, the paper ends with some conclusions.

\section{REPRESENTATION OF THE ATTITUDE}

In our implementation, the quaternion was chosen for the presentation of the attitude as it presents no problem of singularity with respect to Euler angles when the aircraft is vertical (the pitch angle is equal to $-\pi / 2$ ). The unit 
quaternion is composed of a unit vector $\boldsymbol{e}$, called the Euler axis and a rotation angle $\beta$ around this axis:

$$
q=\left[\cos \frac{\beta}{2} e \sin \frac{\beta}{2}\right]^{T}=\left[\begin{array}{ll}
q_{0} & q
\end{array}\right]^{T}
$$

When the system of coordinates $\mathrm{E}^{b}$ attached to the rigid body rotates relatively to the inertial coordinate $\mathrm{E}^{f}$ with an angular velocity $\boldsymbol{\omega}=\left[\begin{array}{lll}\omega_{x} & \omega_{y} & \omega_{z}\end{array}\right]^{T}$, kinematic equations in terms of quaternion are given by:

$$
\dot{q}=\left(\begin{array}{c}
\dot{q}_{0} \\
\dot{\boldsymbol{q}}
\end{array}\right)=\frac{1}{2}\left(\begin{array}{c}
-\boldsymbol{q}^{T} \\
I_{3} q_{0}+\left[\boldsymbol{q}^{\times}\right]
\end{array}\right) \boldsymbol{\omega}=\frac{1}{2} \Xi(q) \boldsymbol{\omega}
$$

In the attitude control, note that $q_{d}$ is the desired attitude and $q$ the real attitude of the rigid body, the error attitude is represented by:

$$
q_{e}=q_{d}^{-1} \otimes q
$$

where $\otimes$ is the multiplication of the quaternion and the conjugate quaternion $q^{-1}$ is the complement rotation of quaternion $q$. In the case where the attitude error is zero, the error quaternion has two possible values: $q_{e}=\left[\begin{array}{ll} \pm 1 & 0^{T}\end{array}\right]^{T}$. This is due to the non bijection of the quaternion with the group $S O(3)$.

\section{CONVERTIBLE PLANE}

In the configuration shown in figure 1 , two counter rotating rotors are placed side by side in the vehicle and inside the wing. The aircraft was properly calibrated so that the center of gravity coincides with the aerodynamic center of the wing and it is at a quarter of the wing's cord. From the model of the convertible plane described above and the kinematic equations (2), the Newton-Euler equations of motion describing the six degrees of freedom of the system can be separated in translational motion $\left(\Sigma_{P}\right)$ and rotational motion $\left(\Sigma_{A}\right)$ :

$$
\begin{gathered}
\Sigma_{P}:\left\{\begin{array}{l}
\dot{\boldsymbol{p}}^{f}=R \boldsymbol{v}^{b} \\
\dot{\boldsymbol{v}}^{b}=R^{T} \boldsymbol{g}^{f}+\frac{1}{\bar{m}} \boldsymbol{F}_{A, T}^{b}-\boldsymbol{\omega} \times \boldsymbol{v}^{b}
\end{array}\right. \\
\Sigma_{A}:\left\{\begin{array}{l}
\dot{q}=\frac{1}{2} \Xi(q) \boldsymbol{\omega} \\
J \dot{\boldsymbol{\omega}}=-\boldsymbol{\omega} \times J \boldsymbol{\omega}+\boldsymbol{\Gamma}_{A, T}
\end{array}\right.
\end{gathered}
$$

where: $q=\left[\begin{array}{ll}q_{0} & \boldsymbol{q}\end{array}\right]^{T}$ is the quaternion vector; $R$ is the rotation matrix from $E_{b}$ to $E_{f} ; \boldsymbol{p}^{f}$ represents the position of the plane in $E_{f} ; \boldsymbol{v}^{b}$ is the linear velocity vector presented in $E_{b} ; \bar{m}=\operatorname{diag}(m) \in \mathbb{R}^{3 \times 3}$ where $m(\simeq 900 g)$ is the weight of the plane; $\boldsymbol{g}^{f}$ is the vector of the gravity acceleration in $E_{f} ; \boldsymbol{\omega}$ is the angular velocity vector; $J \in \mathbb{R}^{3 \times 3}$ represents the inertial matrix of the plane; $\boldsymbol{F}_{A, T}^{b}=\boldsymbol{F}_{A}^{b}+$ $\boldsymbol{F}_{T}^{b}$ is the control vector of the aerodynamic forces and the thrusts expressed in the coordinate $E_{b}$ (see figures 2 and 3); $\boldsymbol{\Gamma}_{A, T}=\boldsymbol{\Gamma}_{A}+\boldsymbol{\Gamma}_{T}$ is the control torque vector.

The forces $\boldsymbol{F}_{T}^{b}$ and the torques $\boldsymbol{\Gamma}_{T}$ generated by the actuators are identical in both the horizontal and vertical flight. As shown in figures 2 and 3, the left motor rotates in the clockwise direction while the right rotates in anticlockwise direction. Each motor produces a force $T_{i}$ parallel to its axis of rotation and a reactive torque $Q_{i}$ opposite to the direction of rotation. The combination of the forces $T_{i}$ and the reactive torques $Q_{i}$ is given by:

$$
\left\{\begin{array}{c}
\boldsymbol{F}_{T}^{b}=\left[\begin{array}{lll}
T_{1}+T_{2} & 0 & 0
\end{array}\right]^{T} \\
\boldsymbol{\Gamma}_{T}=\left[\begin{array}{lll}
Q_{2}-Q_{1} & 0 & \left(T_{2}-T_{1}\right) l_{m}
\end{array}\right]^{T}
\end{array}\right.
$$

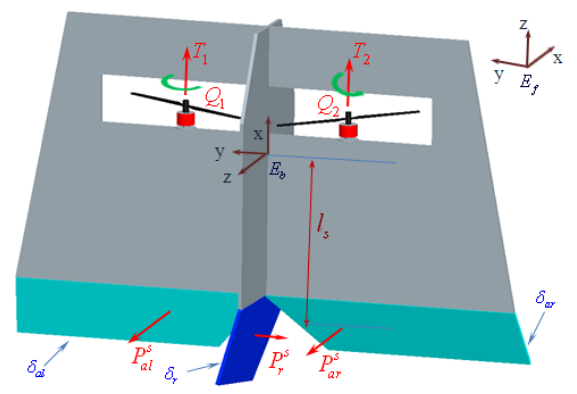

Fig. 2. Vertical flight (helicopter mode)

Since the rotor speed reaches very high values (more than $200 \mathrm{rad} / \mathrm{sec}$ ), we can approximate the forces $T_{i}$ and the reactive torques $Q_{i}$ generated by the motors:

$$
\begin{aligned}
& T_{i}=b_{i} s_{i}^{2} \\
& Q_{i}=k_{i} s_{i}^{2}
\end{aligned}
$$

where $s_{i}$ is the rotational speed of rotor; $b_{i}$ and $k_{i}$ are two positive parameters depending on the density of air, the radius, the shape, the pitch angle of the blade and other factors (Fay [2001]).

The aerodynamic forces $\boldsymbol{F}_{A}^{b}$ and torques $\boldsymbol{\Gamma}_{A}$ depend on the working mode of the plane. In the helicopter mode (see figure 2), the aerodynamic forces and torques are simply calculated:

$$
\left\{\begin{array}{l}
\boldsymbol{F}_{A}^{b}=\left[\begin{array}{ll}
0-P_{r}^{s} P_{a l}^{s}+P_{a r}^{s} & ]^{T} \approx\left[\begin{array}{lll}
0 & 0 & 0
\end{array}\right]^{T} \\
\boldsymbol{\Gamma}_{A}=\left[\left(P_{a l}^{s}-P_{a r}^{s}\right) l_{m}\left(P_{a l}^{s}+P_{a r}^{s}\right) l_{s} P_{r}^{s} l_{s}\right.
\end{array}\right]^{T}
\end{array}\right.
$$

where $P_{i}^{s}=\frac{1}{2} \rho v_{i n}^{2} S_{i}^{s} C_{i}^{P_{\delta}}$ with $i=a l$, ar, $r$ (left aileron, right aileron, and rudder); $\rho$ is the density of air; $C_{i}^{P_{\delta}}=$ $C_{i_{\delta}}^{P} \delta_{i} ; \delta_{i}$ and $S_{i}$ are the deflection angle and the area of control surface; $v_{i n}=\sqrt{\frac{2 T_{i}}{\rho A_{\text {prop }}}}$ is the air inflow velocity created by the propeller (Fay [2001]) and $A_{\text {prop }}=\pi R_{\text {prop }}^{2}$ where $R_{\text {prop }}$ is the propeller's radius.

In horizontal flight, we assume that there is no wind. So the air velocity is equal to the relative velocity of the UAV, but in the opposite direction : $\boldsymbol{V}_{\text {air }}=-\boldsymbol{V}$. The vector of the air velocity creates with the plane an angle of attack $\alpha$ (figure 3). In this mode, the aerodynamic forces and torques are given by:

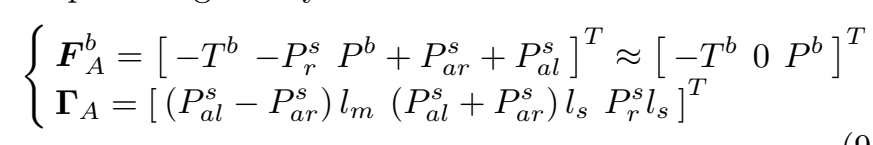

The longitudinal dynamic model of the wing is shown in figure 4 , the aerodynamic lift force $P^{b}$ and drag force $T^{b}$ in the frame $E_{b}$ are calculated from:

$$
\begin{aligned}
& P^{b}=P \cos \alpha+T \sin \alpha \\
& T^{b}=-P \sin \alpha+T \cos \alpha
\end{aligned}
$$

where: $\alpha$ is the angle of attack, $P, T$ are calculated from the aerodynamic formulas in table 1 (see Mc Cormick [1995]), $S$ is the wing's area, the functions $f^{P}(\alpha)$ and $f^{T}(\alpha)$ are the nonlinear functions of the lift and drag coefficients depending on the angle of attack and the wing's profile (figures $5(\mathrm{a})$ and $5(\mathrm{~b}))$ and $C^{T_{0}}$ is the constant coefficient of the parasite drag. 


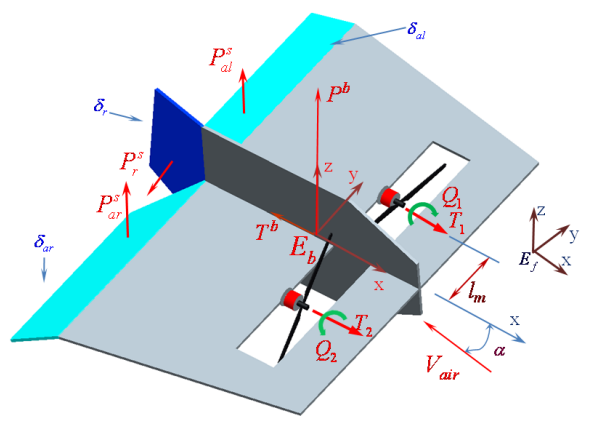

Fig. 3. Horizontal flight (airplane mode)

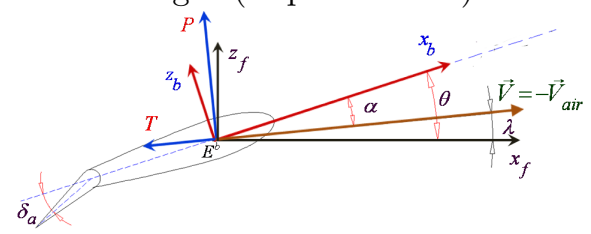

Fig. 4. The longitudinal aerodynamic model

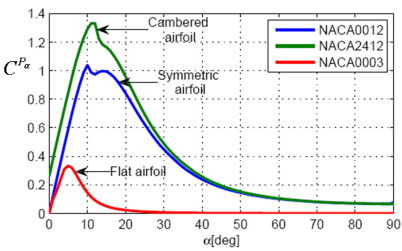

(a) The lift coefficient

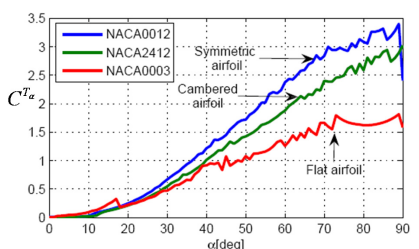

(b) The drag coefficient
Fig. 5. The aerodynamic coefficient depending on the angle of attack and wing's profile

Table 1. Aerodynamic forces

\begin{tabular}{cl}
\hline Lift force & \multicolumn{1}{c}{ Drag force } \\
\hline$P=\frac{1}{2} \rho V^{2} S C^{P}$ & $T=\frac{1}{2} \rho V^{2} S C^{T}$ \\
$C^{P}=f^{P}(\alpha)$ & $C^{T}=C^{T_{\alpha}}+C^{T_{0}}$ \\
& $C^{T_{\alpha}}=f^{T}(\alpha)$ \\
\hline
\end{tabular}

4. CONTROL STRATEGY

\subsection{Control Law}

Altitude Control. The control of the altitude is used when the plane flies vertically but it is not used in the transition and horizontal flight. Extracting the translational motion on the $z$ axis of $E_{f}$ in equation (4) yields:

$$
m \ddot{z}=T \cos \left(\frac{\pi}{2}-\theta\right) \cos \phi-m g
$$

where $T$ is the total thrust force of both motors. A simple PD control can guarantee the convergence of the altitude to the desired altitude $z_{d}$ :

$$
T=T_{1}+T_{2}=\frac{-k_{p}\left(z-z_{d}\right)-k_{d} \dot{z}+m g}{\cos \left(\frac{\pi}{2}-\theta\right) \cos \phi}
$$

But for security reasons and in order to improve the performance, a PID control with saturation is used:

$$
T=\frac{\operatorname{sat}_{T}\left(-k_{p}\left(z-z_{d}\right)-k_{d} \dot{z}-k_{i} \int\left(z-z_{d}\right)\right)+m g}{\cos \left(\frac{\pi}{2}-\theta\right) \cos \phi}
$$

where $\operatorname{sat}_{M}(x)=\{x$ if $|x| \leqslant M ; M \operatorname{sign}(x)$ if $|x|>M\}$ is the classical saturation function, $M$ is the boundary saturation and $\operatorname{sign}(x)$ is the signum function defined as $\operatorname{sign}(x)=\{1$ if $x \geqslant 0 ;-1$ if $x<0\}$.

Attitude Control. In order to control the attitude and to avoid the singularity when the plane flies vertically, the bounded control law with the attitude error calculated by the quaternion error is used. First, we define a scalar function $H\left(q_{0}\right)$ :

Definition 1. $H\left(q_{0}\right)$ is a non-negative function on the interval $q_{0} \in[-1,1]$ and it vanishes only at $q_{0}=-1$ and/or $q_{0}=1$. $H\left(q_{0}\right)$ also satisfies the Lipschitz condition on the interval $q_{0} \in[-1,1]$, that means $\left\|\frac{\partial H}{\partial q_{0}}\right\|_{\infty} \leqslant \gamma$.

Theorem 1. For any function $H\left(q_{0}\right)$ defined above, the following bounded control law globally asymptotically converges the plane to the desired attitude:

$$
\begin{gathered}
\Gamma_{x}=\left(P_{a l}^{s}-P_{a r}^{s}\right) l_{m}=-\operatorname{sat}_{M_{x}}\left(\lambda_{x} \omega_{x}-\rho \frac{\partial H}{\partial q_{e_{0}}} q_{e_{1}}\right) \\
\Gamma_{y}=\left(P_{a l}^{s}+P_{a r}^{s}\right) l_{s}=-\operatorname{sat}_{M_{y}}\left(\lambda_{y} \omega_{y}-\rho \frac{\partial H}{\partial q_{e_{0}}} q_{e_{2}}\right) \\
\Gamma_{z}=\left(T_{2}-T_{1}\right) l_{m}=-\operatorname{sat}_{M_{z}}\left(\lambda_{z} \omega_{z}-\rho \frac{\partial H}{\partial q_{e_{0}}} q_{e_{3}}\right)
\end{gathered}
$$

where $\lambda_{i}, \rho>0 ; M_{i} \geqslant 3 \rho \gamma ; i \in\{x, y, z\}$

This theorem is proved in appendix A.

Table 2 presents some functions $H\left(q_{0}\right)$ with their asymptotic stable equilibrium points (a.s.e.p) and unstable equilibrium points (u.e.p) (Fjellstad et al [1994]).

Table 2. Examples of function $H\left(q_{0}\right)$

\begin{tabular}{ccc}
$H\left(q_{0}\right)$ & a.s.e.p & u.e.p \\
\hline $1-q_{0}$ & $q_{0}=1$ & $q_{0}=-1$ \\
$1+q_{0}$ & $q_{0}=-1$ & $q_{0}=1$ \\
$1-\left|q_{0}\right|$ & $q_{0}= \pm 1$ & \\
$1-\left|q_{0}\right|^{n+1}$ & $q_{0}= \pm 1$ & \\
$\cos \left(\frac{\pi q_{0}}{2}\right)$ & $q_{0}= \pm 1$ & \\
$\cos ^{n}\left(\frac{\pi q_{0}}{2}\right)$ & $q_{0}= \pm 1$ & \\
\hline
\end{tabular}

Since $\left|q_{0}\right| \leqslant 1$ and for simplicity in the embedded control, the function $H\left(q_{0}\right)=1-\left|q_{0}\right|$ was chosen. Its derivative is $\frac{\partial H}{\partial q_{0}}=-\operatorname{sign}\left(q_{0}\right)$. Note that this function does not have unstable equilibrium points, and the control law of the attitude is given by (as in Guerrero et al [2007]):

$$
\begin{aligned}
& \Gamma_{x}=-\operatorname{sat}_{M_{x}}\left(\lambda_{x} \omega_{x}+\rho \operatorname{sign}\left(q_{e_{0}}\right) q_{e_{1}}\right) \\
& \Gamma_{y}=-\operatorname{sat}_{M_{y}}\left(\lambda_{y} \omega_{y}+\rho \operatorname{sign}\left(q_{e_{0}}\right) q_{e_{2}}\right) \\
& \Gamma_{z}=-\operatorname{sat}_{M_{z}}\left(\lambda_{z} \omega_{z}+\rho \operatorname{sign}\left(q_{e_{0}}\right) q_{e_{3}}\right)
\end{aligned}
$$

In order to optimize and improve the performance of the control law, the positive parameters $\lambda_{i}$ and $\rho_{i}$ were calculated according to the pole placement method of bounded control in Johnson et al [2003]. The function $\operatorname{sign}\left(q_{e_{0}}\right)$ ensures that between the two rotations of error angle $\beta_{e}$ and $\left(2 \pi-\beta_{e}\right)$, the smallest angle will be chosen.

\subsection{Trapezoidal Trajectory of Velocity - Servo Control}

In the case when the convertible plane autonomously takes off, lands and makes a transition, the controller requires the next desired position of the altitude or the attitude (pitch angle in our case). Then a trajectory generation algorithm must be used for optimum motion control. The 


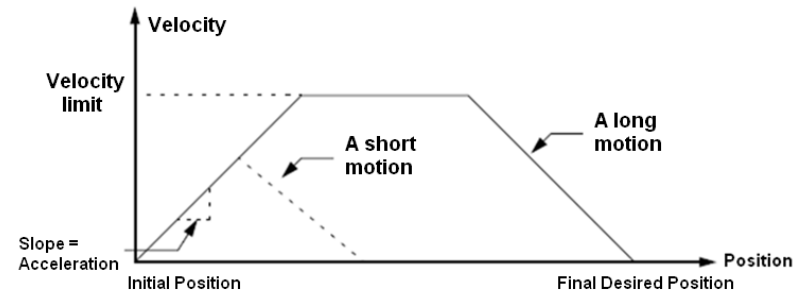

Fig. 6. Trapezoidal Trajectory of Velocity

trajectory generation may not be used by simply forcing the final desired position. However when the final desired position is reached, the required deceleration to stop the vehicle will be very high. Since the vehicle can not achieve such a high deceleration because it flies in the very low friction environment (air), it will certainly overpass the final position. Therefore the vehicle oscillates around the final position before reaching it. The overshooting and oscillation will slow the final objective and have dangerous consequences in the case of autonomous transition and landing. In order to produce a smooth motion, we need a motion profile algorithm that controls the speed and acceleration. A linear piecewise velocity trajectory is implemented: the velocity is incremented by a constant acceleration value until a specified maximum velocity is reached. The maximum velocity is maintained for a required amount of time and then decreased by the same acceleration (deceleration) value until zero velocity is attained (see Figure 6). A movement distance, velocity limit, and acceleration value are required to execute the profile. From these data, the trajectory generator will produce trapezoidal shaped velocity curves for a long movement and triangular curves for a short movement where maximum velocity was not reached. The trapezoidal profile of velocity must be within the velocity profile of $\nu_{\max }$ (maximum physical velocity) and $\dot{\nu}_{\max }$ (maximum acceleration). The control law of altitude and attitude above will force the vehicle to track the new controlled positions created by the trajectory generator in order to realize the process of take off, landing and transition.

\section{RESULTS}

\subsection{Simulation Results}

In this section, some simulation results from MatlabSimulink simulator are presented to demonstrate the performance of the control strategy. Initially, the plane takes off automatically up to $2 \mathrm{~m}$ (figure 7 ), the altitude velocity has a trapezoidal form, it accelerates and reduces the speed when the vehicle reaches $2 \mathrm{~m}$ (figure 8 , period (1)). Then the plane makes the transition (period (2)) at second 3.5, the pitch angle varies from $-90^{\circ}$ to $-2^{\circ}$. After the transition, the plane accelerates to reach a certain speed. Its velocity $v_{x}$ increases rapidly and converges to a constant value due to the friction drag. That means at this time, the longitudinal acceleration of the plane is zero. In figure 11 , we can notice that the angle of attack also converges to a constant value. Despite this small value (approximately $\left.1.5^{\circ}\right)$, it is enough to create the lift force thanks to the high speed of the plane (note that nominal airspeed is about $13 \mathrm{~m} / \mathrm{s}$ ). It flies horizontally about $5 \mathrm{~s}$ (period (3)) and then makes the transition to the vertical at second 11

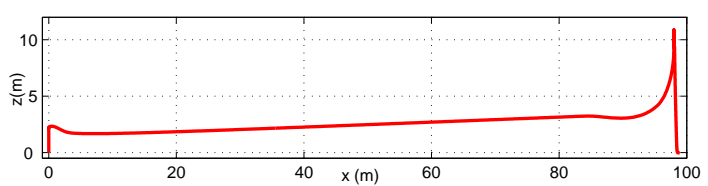

Fig. 7. Longitudinal flight path

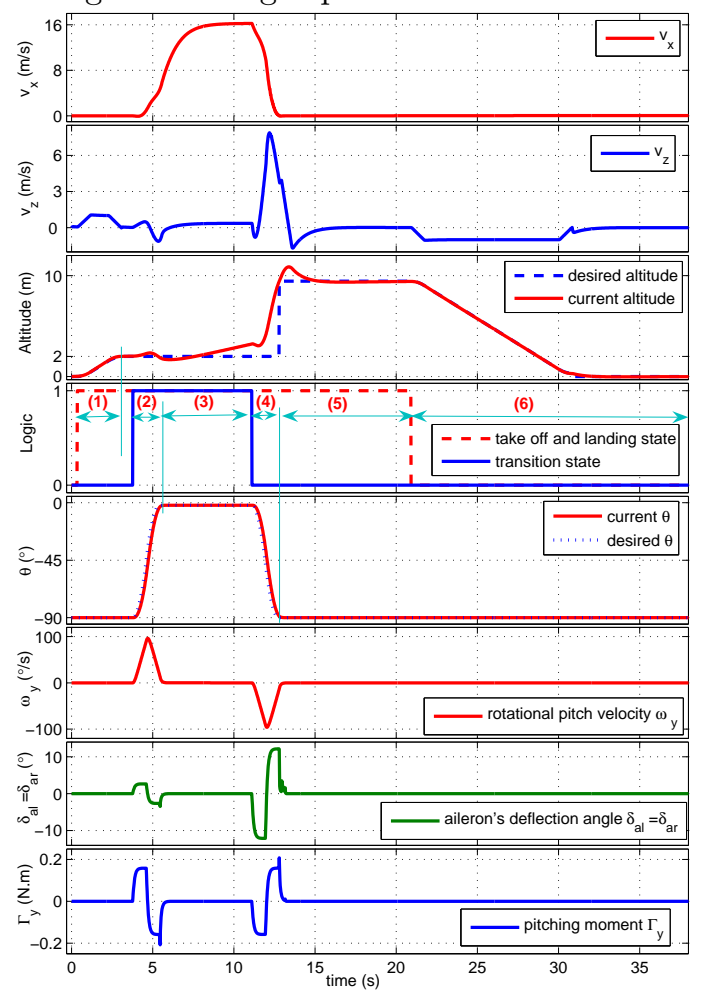

Fig. 8. States of the plane during the period of flight

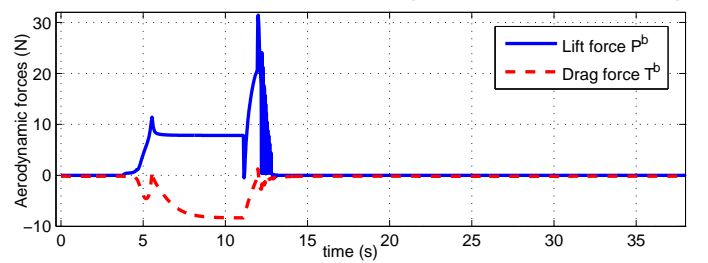

Fig. 9. Aerodynamic force in $E_{b}$ axis

(period (4)). After the transition, it descends and lands at second 21 (period (5) and (6)).

Note that the control law of the altitude is inactivated during the horizontal flight and during the transition phase. The profile of the altitude velocity $v_{z}$ is trapezoidal in the periods of take off (1) and landing (6) as much as the profile of the pitch rotational velocity $\omega_{y}$ has a form of a triangle (figure 8). There is no overshooting in both cases. On the longitudinal flight path (figure 7), the plane loses the altitude in two cases: the first after the transition to the horizontal because its slow speed does not allow to create the needed lift force; the second before the transition to the vertical due to the deceleration of the plane.

\subsection{Experimental Results}

Platform - Embedded Control System. The convertible airplane has been completely designed and constructed at Heudiasyc laboratory (see Figure 1). It has a total weight 


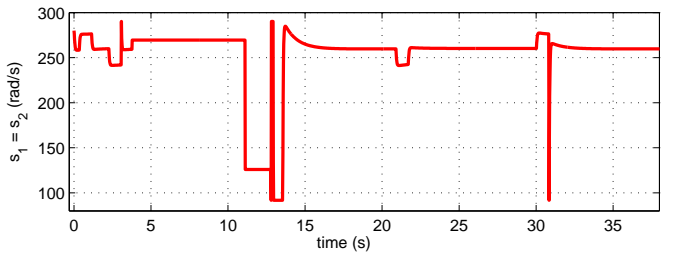

Fig. 10. Speed of each rotor
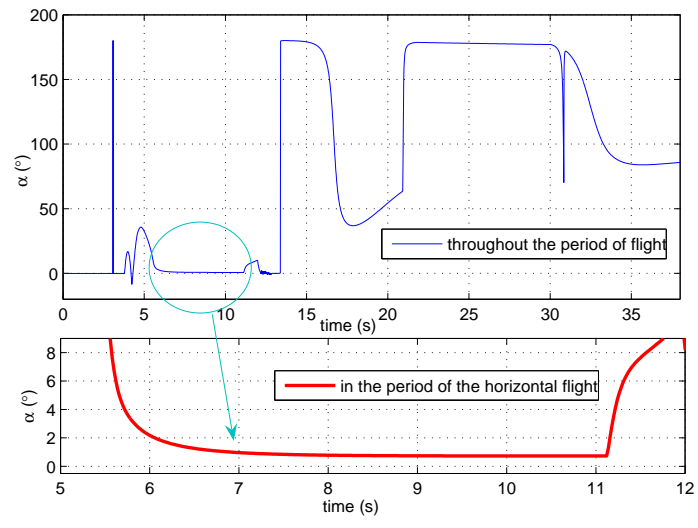

Fig. 11. Angle of attack

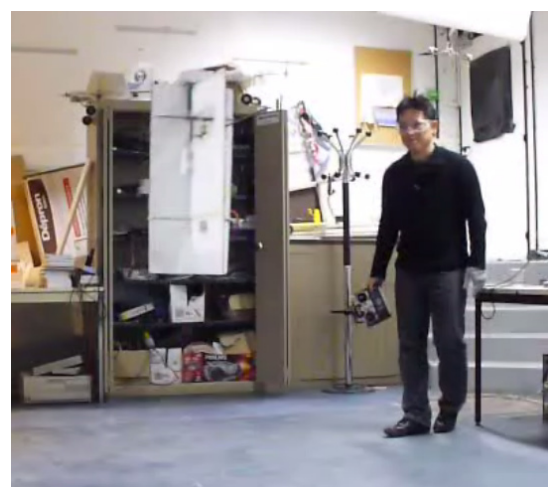

Fig. 12. Vehicle hovering during the vertical flight mode

of about $900 \mathrm{~g}$ and uses one $2200 \mathrm{mAh}$ LiPo battery. Flight endurance is satisfactory for the chosen configuration since we achieve approximately 7 minutes in vertical flight.

An Inertial Measurement Unit (Microstrain, 3DM-GX325 ) is used for obtaining the attitude. The gyro range is $300^{\circ} / \mathrm{s}$, which is enough to handle the transition from vertical to horizontal flight and vice versa. A Digital Signal Controller (Microchip, dsPIC33FJ256GP710) was selected to implement the Embedded Control System, which includes acquiring attitude data from the IMU, reading the control inputs from the $\mathrm{R} / \mathrm{C}$ receiver, implementing the control algorithm, generating the corresponding pulse width modulated (PWM) servo signals to control the amount of aileron and rudder deflection and transmitting flight parameters to a ground station via the serial wireless transmission (XBee-Pro). The measurement used for the altitude is obtained from the low cost ultrasonic sensor (SRF10) which has a range of $10 \mathrm{~cm}$ to $3 \mathrm{~m}$. Figure 12 shows the prototype during a vertical flight when the attitude and the altitude are controlled autonomously.

Vertical Flight Mode Test. In this test, the inputs of the system are roll, pitch and yaw angles given by the opera-

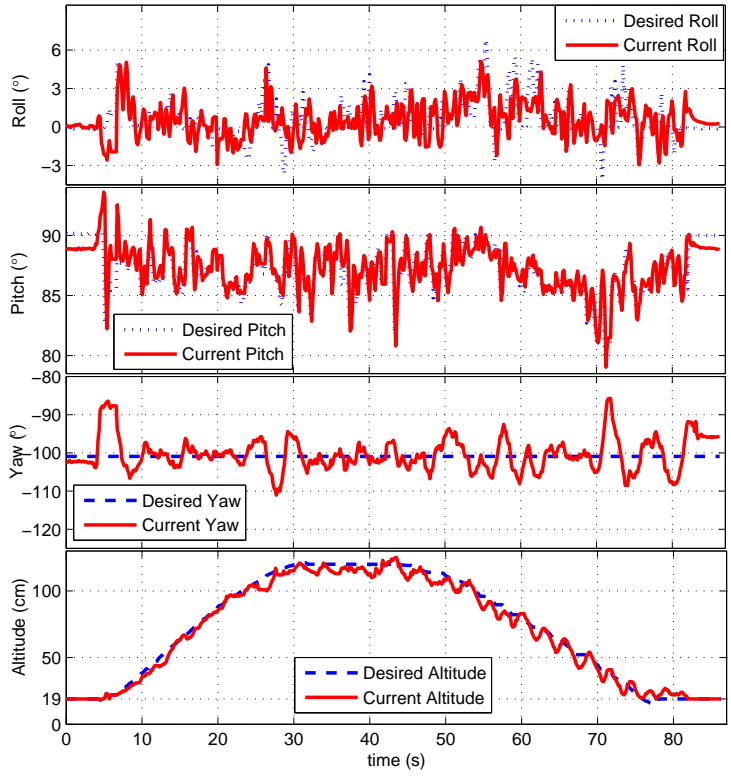

Fig. 13. Vertical Take Off and Landing Flight Test

tors $\mathrm{R} / \mathrm{C}$ transmitter. The desired altitude in take off and landing phases is given by the trajectory generator and the global thrust is generated by the altitude control. Figure 13 represents the results of the attitude and altitude control algorithm during a vertical flight. The presented attitude is recalculated from the current quaternion vector. The pitch angle varies around $87.5^{\circ}$ because of the mechanical calibration error of the plane. However, the vehicle remains stable and follows the desired attitude. The altitude varies respect to the desired one, automatic take off and landing have been tested with success. Note that $19 \mathrm{~cm}$ is the initial altitude of the ultrasonic sensor with respect to the ground since the ultrasonic sensor is placed at this distance from the bottom of the plane. One complete video of vertical take off and landing and vertical flight (including "handsoff", torque perturbation, $360^{\circ}$ yaw rotation tests) was uploaded on the site: http://www.youtube.com/watch? $\mathrm{v}=7 \mathrm{D} 0 \mathrm{qSMTF} q \mathrm{q}$

Transition and Horizontal Flight Mode Test. The tests for transition and horizontal flights are in progress. The results will be obtained soon.

\section{CONCLUSIONS AND FUTURE WORKS}

In this work, we have presented the dynamic model of a convertible plane and a simple control strategy to achieve a longitudinal flight path. The obtained simulation results demonstrate that the proposed control strategy could be implemented in real experiments. The control law is simple and suitable for embedded applications, it does not require a high computational cost for control loop. The proposed approach is currently implemented on the platform to verify the practical applicability of the control strategy. The vertical flight mode has been validated and the other experimental phases are in progress.

\section{ACKNOWLEDGEMENTS}

This work has been supported by the French Armament Procurement Agency (DGA), the French National Center 
for Scientific Research (CNRS) and the project HORUS from the ANR ARPEGE.

\section{REFERENCES}

J. F. Guerrero, A. Hably, N. Marchand, and S. Lesecq. Bounded attitude stabilization : Application on fourrotor helicopter. In 2007 IEEE International Conference on Robotics and Automation, April 2007.

W. E. Green and P. Y. Oh. A Hybrid MAV for Ingress and Egress of Urban Environments, IEEE Transactions On Robotics, Vol. 25, No. 2, April 2009

R. Lozano, editor. Unmanned Aerial Vehicles. John Wiley, 2010.

B. W. Mc Cormick. Aerodynamics Aeronautics and Flight Mechanics, John Wiley and Sons Inc, 1995

M. V. Cook. Flight Dynamics Principles: A Linear Systems Approach to Aircraft Stability and Control, Elsevier, 2007

G. Fay. Derivation of the aerodynamic forces for the mesicopter simulation. Technical Report, Stanford University, USA, 2001.

E. N. Johnson and S. K. Kannan. Nested saturation with guaranteed real poles, In American Control Conference 2003, volume 1, pages 497502, 2003

A. R. Teel. Global stabilization and restricted tracking for multiple integrators with bounded controls, Systems and Control Letters, 18:165-171, 1992.

R. H. Stone. Control architecture for a tail-sitter unmanned air vehicle. In 5th Asian Control Conference, pages 736744, 2004.

N. B. Knoebel and T. W. McLain. Adaptive Quaternion Control of a Miniature Tailsitter UAV, American Control Conference, Westin Seattle Hotel, Seattle, Washington, USA, pages 2340-2345, 2008.

B. Bataillé, D. Poinsot, J.-M. Moschetta, C. Brard, A. Piquereau. Development of a VTOL Mini UAV for MultiTasking Missions, International Powered Lift Conference, London 2008.

O.-E. Fjellstad and T. I. Fossen. Quaternion Feedback Regulation of Underwater Vehicles, In Proceedings of the 3rd IEEE Conference on Control Application, 1994

\section{Appendix A. PROOF OF THEOREM 1}

Proof. Let us define the Lyapunov function:

$$
V=\frac{1}{2} \boldsymbol{\omega}^{T} J \boldsymbol{\omega}+2 \kappa H\left(q_{e_{0}}\right) \geqslant 0
$$

where $\kappa>0$. Differentiating $V$ with respect to time yields:

$$
\begin{gathered}
\dot{V}=\boldsymbol{\omega}^{T} J \dot{\boldsymbol{\omega}}+2 \kappa \frac{\partial H}{\partial q_{e_{0}}} \dot{q}_{e_{0}} \\
\dot{V}=\boldsymbol{\omega}^{T}[-\boldsymbol{\omega} \times J \boldsymbol{\omega}+\boldsymbol{\Gamma}]+2 \kappa \frac{\partial H}{\partial q_{e_{0}}}\left(-\frac{1}{2} \boldsymbol{q}_{e}^{T} \boldsymbol{\omega}\right) \\
\dot{V}=\boldsymbol{\omega}^{T} \boldsymbol{\Gamma}-\kappa \frac{\partial H}{\partial q_{e_{0}}} \boldsymbol{q}_{e}^{T} \boldsymbol{\omega} \\
+\underbrace{\underbrace{\omega_{x} \Gamma_{x}-\kappa \frac{\partial H}{\partial q_{e_{0}}} q_{e_{1}} \omega_{x}}_{\dot{V}_{2}}}_{\dot{V}_{1}}+\underbrace{\omega_{y} \Gamma_{y}-\kappa \frac{\partial H}{\partial q_{e_{0}}} q_{e_{3}} \omega_{z}}_{\dot{V}_{3}} q_{e_{2} \omega_{y}}
\end{gathered}
$$

Because $\dot{V}$ is the sum of three terms $\left(\dot{V}_{1}, \dot{V}_{2}, \dot{V}_{3}\right)$, then we first analyse the term:

$$
\dot{V}_{1}=\omega_{x} \Gamma_{x}-\kappa \frac{\partial H}{\partial q_{e_{0}}} q_{e_{1}} \omega_{x}
$$

Applying the bounded control law (14), we have:

$$
\dot{V}_{1}=-\omega_{x} \operatorname{sat}_{M_{x}}\left[\lambda_{x} \omega_{x}-\rho \frac{\partial H}{\partial q_{e_{0}}} q_{e_{1}}\right]-\kappa \frac{\partial H}{\partial q_{e_{0}}} q_{e_{1}} \omega_{x}
$$

We assume that:

$$
\left|\lambda_{x} \omega_{x}\right|>2 \rho \gamma \geqslant 2 \rho\left\|\frac{\partial H}{\partial q_{e_{0}}}\right\|_{\infty} \Leftrightarrow\left|\omega_{x}\right|>\frac{2 \rho \gamma}{\lambda_{x}}
$$

then we have $\left|\lambda_{x} \omega_{x}-\rho \frac{\partial H}{\partial q_{e_{0}}} q_{e_{1}}\right| \geqslant \rho \gamma+\varepsilon$ (with $\varepsilon>0$ ) and $\left(\lambda_{x} \omega_{x}-\rho \frac{\partial H}{\partial q_{e_{0}}} q_{e_{1}}\right)$ has the same sign as $\omega_{x}$. Then:

$$
\begin{aligned}
& \dot{V}_{1}=-\omega_{x} \operatorname{sat}_{M_{x}}\left[\lambda_{x} \omega_{x}-\rho \frac{\partial H}{\partial q_{e_{0}}} q_{e_{1}}\right]-\kappa \frac{\partial H}{\partial q_{e_{0}}} q_{e_{1}} \omega_{x} \\
& \leqslant-\left|\omega_{x}\right| \operatorname{sat}_{M_{x}}[\rho \gamma+\varepsilon]+\kappa \gamma\left|\omega_{x}\right|
\end{aligned}
$$

By choosing:

$$
\kappa \gamma<\min \left(M_{x}, \rho \gamma+\varepsilon\right) \Leftrightarrow \kappa<\frac{\min \left(M_{x}, \rho \gamma+\varepsilon\right)}{\gamma}
$$

we can assure the decrease of $V_{1}$ because $\dot{V}_{1}<0$. Consequently, $\omega_{x}$ enters in the set $\Phi_{x}=\left\{\omega_{x}:\left|\omega_{x}\right| \leqslant \frac{2 \rho \gamma}{\lambda_{x}}\right\}$ in finite time $t_{1}$ and remains in it thereafter. In this case, $\left(\lambda_{x} \omega_{x}-\rho \frac{\partial H}{\partial q_{e_{0}}} q_{e_{1}}\right) \in[-3 \rho \gamma, 3 \rho \gamma]$. Let's choose $M_{x}$ which satisfies the following condition: $M_{x} \geqslant 3 \rho \gamma$. Then for time $t_{2}>t_{1}$, the argument $s a t_{M_{x}}$ will be bounded as follows: $\left|\lambda_{x} \omega_{x}-\rho \frac{\partial H}{\partial q_{e_{0}}} q_{e_{1}}\right| \leqslant 3 \rho \gamma \leqslant M_{x}$ and then we have a condition for $\kappa$ from A.10:

$$
\kappa<\frac{\rho \gamma+\varepsilon}{\gamma}=\rho+\frac{\varepsilon}{\gamma}
$$

Consequently, sat $_{M_{x}}$ operates in a linear region:

$$
\Gamma_{x}=-\lambda_{x} \omega_{x}-\rho \frac{\partial H}{\partial q_{e_{0}}} q_{e_{1}}
$$

and:

$$
\begin{gathered}
\dot{V}_{1}=-\omega_{x}\left(\lambda_{x} \omega_{x}-\rho \frac{\partial H}{\partial q_{e_{0}}} q_{e_{1}}\right)-\kappa \frac{\partial H}{\partial q_{e_{0}}} q_{e_{1}} \omega_{x} \\
\dot{V}_{1}=-\lambda_{x} \omega_{x}^{2}+\rho \frac{\partial H}{\partial q_{e_{0}}} q_{e_{1}} \omega_{x}-\kappa \frac{\partial H}{\partial q_{e_{0}}} q_{e_{1}} \omega_{x}
\end{gathered}
$$

By choosing $\kappa=\rho$ satisfying condition A.11, we have: $\dot{V}_{1}=-\lambda_{x} \omega_{x}^{2} \leqslant 0$ The same argument is applied to $\dot{V}_{2}$ and $\dot{V}_{3}$, and $\dot{V}$ becomes:

$$
\dot{V}=\dot{V}_{1}+\dot{V}_{2}+\dot{V}_{3}=-\left(\lambda_{x} \omega_{x}^{2}+\lambda_{y} \omega_{y}^{2}+\lambda_{z} \omega_{z}^{2}\right) \leqslant 0
$$

In order to complete the proof, LaSalle Invariance Principle is invoked. All the trajectories converge to the largest invariant set $\bar{\Omega}$ in $\Omega=\left\{\left(\boldsymbol{q}_{e}, \boldsymbol{\omega}\right): \dot{V}=0\right\}=$ $\left\{\left(\boldsymbol{q}_{e}, \boldsymbol{\omega}\right): \boldsymbol{\omega}=0\right\}$. In the invariant set, $J \dot{\boldsymbol{\omega}}=-\boldsymbol{\omega} \times J \boldsymbol{\omega}+$ $\boldsymbol{\Gamma}=\rho \frac{\partial H}{\partial q_{e_{0}}}\left[\begin{array}{lll}q_{e_{1}} & q_{e_{2}} & q_{e_{3}}\end{array}\right]=0$. That is, $\bar{\Omega}$ is reduced to the origin: $\boldsymbol{q}_{e}=\boldsymbol{\omega}=0$. This ends the demonstration of the asymptotic stability of the closed-loop system. 